\title{
REDUCED ORDER TRANSMISSION LINE MODELING FOR IMPROVED EFFICIENCY IN THE CALCULATION OF ELECTROMAGNETIC TRANSIENTS
}

\author{
A. Oguz Soysal* \\ Adam Semlyen \\ Department of Electrical and Computer Engineering \\ University of Toronto \\ Toronto, Ontario, Canada, M5S 1A4 \\ * On leave from Karadeniz Technical University, Trabzon, Turkey
}

\begin{abstract}
A direct method based on the linear least squares approach is applied for obtaining reduced order approximations of the propagation and characteristic admittance transfer functions of transmission lines for use in calculation of transients. The practical level of the acceptable error is discussed in terms of the precision of the frequency domain input data. The proposed approach provides satisfactory accuracy with a small number of real and/or complex poles. The low order approximation could improve the efficiency of the EMTP by reducing the computation time.
\end{abstract}

Keywords: Electromagnetic transients, Transmission line modeling, Frequency response, Transfer function realization, Estimation.

\section{INTRODUCTION}

The standard EMTP (Electro-Magnetic Transients Program) is based on the direct time domain solution of the ordinary differential equations (ODEs) representing the system components, by taking into account the delay time due to the wave propagation on transmission lines [1],[2]. Time domain analysis permits the simulation of various operating conditions in a straightforward way. However, due to the frequency dependent parameters resulting from complex field phenomena in the ground and inside the conductors, transmission lines are first modeled in the frequency domain. The state equation approximation of transmission lines requires an intermediate stage of rational function fitting to the frequency domain data obtained from the line geometry.

The transient analysis of a transmission line is based on two frequency domain complex functions, the propagation transfer function $H_{P}$ and the characteristic admittance function $Y_{C}$. In conventional EMTP calculations. both $H_{P}$ and $Y_{C}$ are approximated by rational functions with real poles [3]-[6]. The order of these functions depends on the line geometry, the considered frequency range and the required accuracy. It is possible to obtain a sufficiently accurate fitting with a large number of real poles. The order of the identified rational functions, however, determines the number of the state equations to be solved to obtain the time domain responses. In the case of complex line arrangements such as three phase, multiple circuit lines, high order accurate fitting would result in a very large number of differential equations. In [6] Marti has shown that lower order fitting may be used by a suitable compromise between the accuracy of the simulation and simplicity of the model used.
In this paper the rational functions corresponding to $H_{P}$ and $Y_{C}$ are estimated by means of a direct method based on the solution of a linear least squares problem using singular value decomposition (SVD) as shown in [7]. An appropriate weighting is applied to increase the accuracy of the fitting at low and middle frequency range data. Since the method is not limited to real poles only, it makes possible to achieve an accurate fitting with a smaller number of real and/or complex conjugate poles. Once the transfer function is obtained as a sum of partial fractions, the state equations with real matrices can be written in a canonical form in terms of the poles and the pole strengths.

The proposed methodology provides high accuracy with low model orders. It results in considerable savings in computation time, thus improving the efficiency of EMTP calculations. This improvement may become particularly significant in the analysis of three phase transients in networks with multiple circuit transnission lines.

\section{APPROXIMATION OF \\ TRANSMISSION LINE TRANSFER FUNCTIONS}

The line models currently used in EMTP studies require a rational function approximation of the propagation and characteristic admittance (or impedance) transfer functions, for each mode $i$,

$$
\begin{gathered}
H_{P, i}(\mathrm{j} \omega)=\exp \left(-\sqrt{Z_{i}(\mathrm{j} \omega) Y_{i}(\mathrm{j} \omega) l}\right) \\
Y_{C, i}(\mathrm{j} \omega)=\sqrt{Y_{i}(\mathrm{j} \omega) / Z_{i}(\mathrm{j} \omega)}
\end{gathered}
$$

where $l$ is the line length and $Z_{i}(\mathrm{j} \omega), Y_{i}(\mathrm{j} \omega)$ are the line parameters for mode $i$, determined in terms of line geometry by using basic field relations together with Carson's formulae or with the concept of complex penetration depth [3]-[5].

\section{The Basic Estimation Methodology}

The scalar estimation methodology, used for rational function approximation of both $H_{P}$ and $Y_{C}$, is described in detail in [7] and is reviewed succinctly in the following. In the general case, the transfer function can be written as

$$
H(s)=\frac{a_{0}+a_{1} s+a_{2} s^{2}+\cdots+a_{\mu} s^{\mu}}{b_{0}+b_{1} s+b_{2} s^{2}+\cdots+b_{v} s^{v}}
$$

If $H(s)$ is strictly proper, then $\mu<v$, normally, $\mu=v-1$. Assuming that the frequency domain complex output data is known for discrete frequency values $j \omega_{k}$, we obtain a set of overdetermined linear equations

$$
\mathbf{A x}=\mathbf{b}
$$

by equating the real and imaginary parts of (3) for $s=j \omega_{k}$. In (4), A is an $m \times n$ matrix, where $n$ is the number of parameters in (3) to be identified, and $m(\geq n)$ the number of real observations. The vector of unknown parameters $\mathbf{x}$ can be determined in the least squares sense by using an appropriate solution technique. However, due to the poor numerical condition of the problem, the use of the normal equations is not recommended. As discussed in [7], the condition of A can be significantly improved by column scaling and thell (4) can
93 SM 510-8 PWRS A paper recommended and approved by the IEEE Power System Engineering Committee of July 18-22, 1993. Manuscript submitted 31, 1992; made available for printing April 26, 1993

PRINTED IN USA 
be solved by using Singular Value Decomposition (SVD). The obtained least squares solution minimizes the euclidian norm of the residual

$$
\varepsilon=\|\mathbf{A x}-\mathbf{b}\|
$$

After the coefficients of (3) are identified, the transfer function can be written in terms of partial fractions as

$$
H(s)=\sum_{i=1}^{\mathrm{v} / 2}\left[\frac{\alpha_{i}+\mathrm{j} \beta_{i}}{s-\sigma_{i}-\mathrm{j} \omega_{i}}+\frac{\alpha_{i}-\mathrm{j} \beta_{i}}{s-\sigma_{i}+\mathrm{j} \omega_{i}}\right)
$$

Here, for simplicity, it is assumed that all the poles are complex. Real poles can be considered as a special case of the complex formulation. The new parameters, $\alpha, \beta, \sigma$, and $\omega$, are then refined by an iterative process with restriction on $\sigma$, termed Gauss-Newton Improvement, to impede obtaining unstable poles. This restriction has of course no significance in our application where both transfer functions are always intrinsically stable.

\section{Approximation of the Propagation Transfer Function}

The effect of propagation of a (current or voltage) wave along a transmission line is described by the equation

$$
W^{\prime}=-H_{P} W_{\text {far }}{ }^{\prime \prime}
$$

where $W^{\prime}$ and $W_{\text {far }}{ }^{\prime \prime}$ represent the incident and reflected waves, respectively. The propagation equation (7) involves inherently the propagation delay

$$
\tau=\frac{l}{c}
$$

$c$ being the velocity of light. Usually, this delay is taken into account in the time domain formulation. Therefore, its effect in the frequency domain can be compensated by decomposing $H_{p}$ into two factors, as shown in [4], resulting in

$$
H_{P}^{\prime}=H_{P} e^{j \omega \tau}
$$

$H_{p}^{\prime}$ is then evaluated for $m$ discrete frequency values $\omega_{k}$ to obtain the frequency domain data to be used as input to the estimation process. This procedure has been termed backwinding in [4].

While $H_{P}$ depends on the line length and geometry, its general shape is as shown for an example line in Figure 2. The magnitude at the lower frequency range remains near unity. The transmission line behaves as a low-pass filter, since the components beyond a certain cut-off frequency are fully choked off along the line.

Practical examples have shown that the estimation procedure used provides very good accuracy in the high frequency range for $H_{p}$. At lower frequencies, the error may be slightly bigger with a reduced order fitting. It is always possible to force the estimated rational function to satisfy the boundary condition at $\omega=0$, by taking $a_{0}=b_{0}$ in (3), which means reducing the number of unknowns by 1 in (4), and therefore, deleting the first column of $A$. This extra condition, however, reduces the degree of freedom of the least squares fitting, resulting in bigger errors at the high frequency range. Moreover, it has been observed that the magnitude of $H_{P}$ may become bigger than unity at low frequencies when the zero frequency boundary condition is introduced. While the solution is mathematically acceptable, it may result in large errors in the calculation of transients.

In order to improve the accuracy of the fitting at the low frequency range, an appropriate weighting can be applied to the linear equations (4). This could be particularly important in the case of short lines (of a few kilometers or less) where the dominant frequencies of the transient may be located in the initial flat region. It is practically meaningful to choose the weighting function in terms of the observation values ( $b$ in (4) ) since the errors on bigger values of $H_{P}$ are more important in the computation of transients. Practical examples have shown that weighting by the squares of the observation values reduces significantly the fitting error. Both sides of (4) are therefore left-multiplied by the diagonal matrix

$$
\mathbf{D}=\operatorname{diag}\left\{b_{i}^{2}\right\}
$$

\section{Approximation of the Characteristic Admittance Function}

The voltages and currents at the line ends are related by the frequency domain terminal equation:

$$
I_{Y}=Y_{C} V
$$

The characteristic adnittance $Y_{C}$ of a transmission line tends to an asymptotic value $Y_{\infty}$ at infinite frequency, i.e. $Y_{C}$ is proper. In the basic estimation methodology used, it is assumed that the system is strictly proper. It is, therefore, necessary to preprocess $Y_{C}$ by decomposing it as shown below:

$$
I_{Y}=\left(Y_{C}{ }^{\prime}+Y_{\infty}\right) V
$$

Now $Y_{C}{ }^{\prime}$ can be approximated by a rational function using the methodology described in [7]. The weighting shown above for the approximation of $H_{P}$ is also used to improve the accuracy in fitting of $Y_{C}{ }^{\prime}$. The asymptotic value, $Y_{\infty}$, is taken into account in the time domain formulation of the output variable $i_{Y}(t)$.

\section{ACCURACY OF THE REALIZATION}

The order of the rational functions estimated for $H_{P}$ and $Y_{C}$ depends on the required accuracy. Since the order is not known in advance, it is convenient to start with order 1 , and increase it gradually until the required accuracy is achieved. To apply this strategy, an error criterion and error limits should be appropriately defined.

The frequency domain error of the transfer function approximation is defined here as the RMS of the absolute values of the complex differences between the input data and the estimated rational function. Clearly, this reflects both the errors of magnitude and angle. However, the main purpose of the approximation is to obtain a model for time domain calculations. The final evaluation of the accuracy should therefore be made in the time domain, for example by means of the step response.

The appropriate choice of an acceptable error level is essential for obtaining the optimum model order. The precision of the estimation process depends obviously on the accuracy of the available input data. The geometric dimensions and conductor properties are generally well defined along the line. The resistivity of the earth. however, depends on various factors, and can not be accurately determined. Moreover, its value may also vary due to the external conditions. In practice, the variation of the ground resistivity in a fairly wide range introduces an uncertainty in the calculation of the parameters and consequently in the frequency domain data.

The effects of the ground resistivity on the propagation and characteristic admittance transfer functions are exanined for the overhead line configuration shown in Figure 1. The variations of $H_{P}$

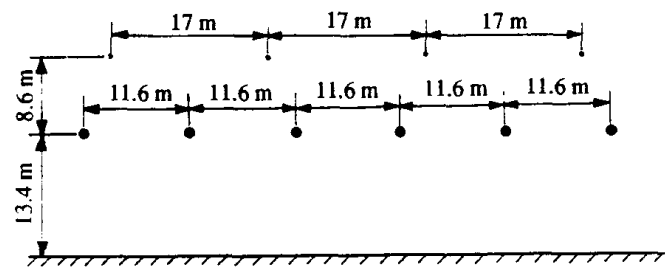

Figure 1 Configuration of the overhead line. 
computed with ground resistivities of $50 \Omega \mathrm{m}$ and $150 \Omega \mathrm{m}$, differing by $\pm 50 \Omega \mathrm{m}$ from the base value of $100 \Omega \mathrm{m}$, are shown for different modes in Figure 2. Note that Figure 2 and the following Figure 3 illustrate only the effect of variations of the ground resistivity with "exact" calculations; the effect of errors due to fitting is shown in Figure 4.

Figure 2 shows that the change in the ground resistivity has shifted the frequency response curves and has slightly changed the slope. This has resulted in a time delay in the step responses given in Figure 3. In the line modes the effect of the frequency domain error due to the variation of the ground resistivity is almost invisible in the step response. The ground mode (no. 6 in the figures) is, however, more affected in the time domain although the frequency domain error is small. This apparently is the consequence of the slower increase of the step response corresponding to the ground mode.
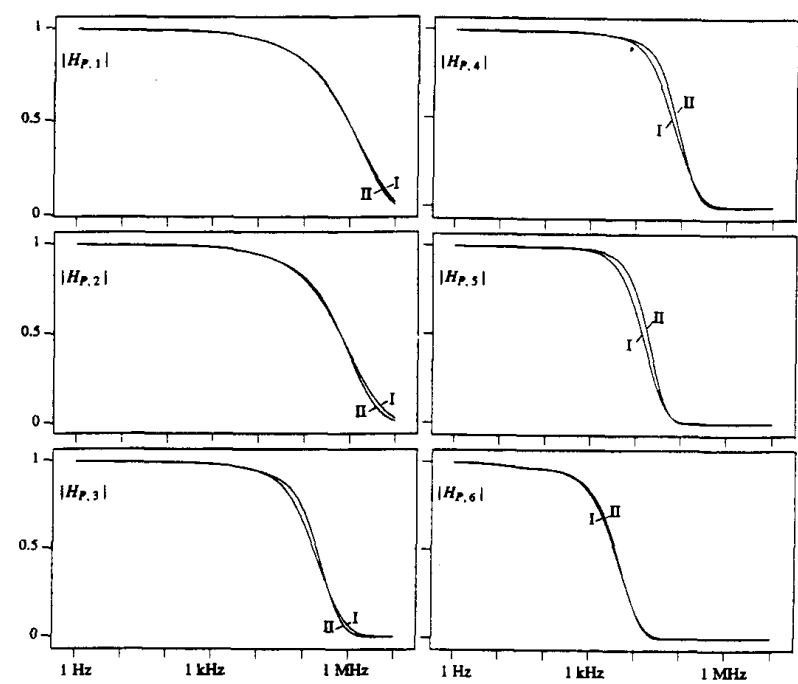

Figure 2 Frequency domain variation of the modal propagation functions $H_{P}$ for $\rho=50 \Omega \mathrm{m}$ (I), and $\rho=150 \Omega \mathrm{m}$ (II).
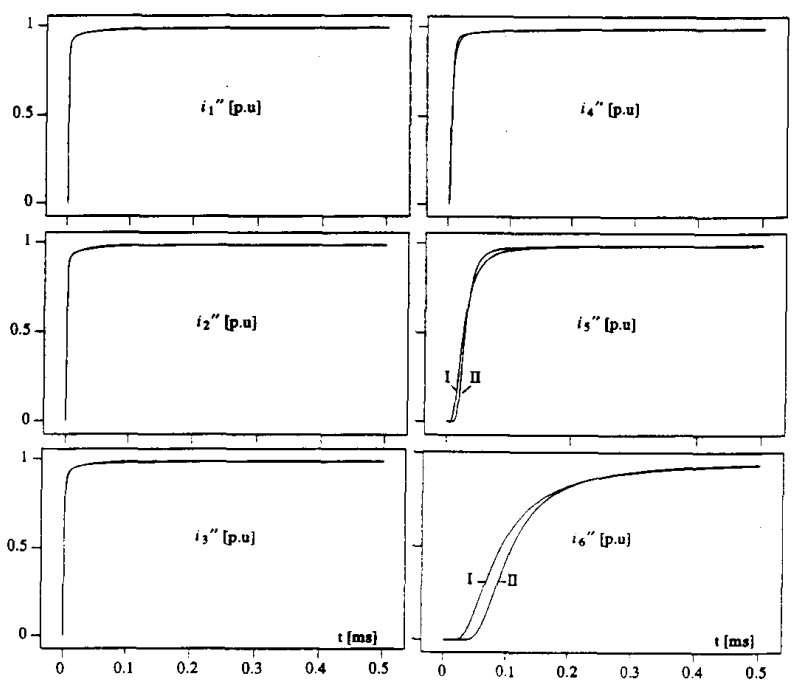

Figure 3 Step responses of modal propagation functions for $\rho=50 \Omega \mathrm{m}(\mathrm{I})$, and $\rho=150 \Omega \mathrm{m}$ (II).
It can be concluded that a relatively bigger fitting error may be acceptable on the slope of the frequency responses, since the data corresponding to this portion can not be defined precisely in practice. The low frequency portion of the curve is only relevant for a very long integration time. In the calculation of fast transient phenomena, such as in most cases that arise in the analysis of switching or lightning surges, high accuracy may therefore not be needed in the very low frequency range.

The fitting at the critical frequency range where $H_{P}$ is very small (viz. at the lower bend of the curve) can be improved by backwinding with an appropriate additional delay, $\Delta \tau$ [3],[4]. This would mean for example in the case of step responses for modes 5 and 6 of Figure 3 that, due to the shift of origin by $\Delta \tau$, the initial toe portion of the responses is removed and the frontal slopes of these curves start from the origin. Hence, obviously, fewer exponentials are needed for their fitting. Equivalently, after backwinding, the approximation for modes 5 and 6 of Figure 2 can be obtained by lower order rational polynomials. Since, however, $\Delta \tau$ is not known in advance, by not inplementing any backwinding the fitting errors with low order approximation may produce small fluctuations at the beginning of the step response. Although these fluctuations have no practical significance, they can be completely removed by taking into account the additional delay in the time domain computations. Therefore, implementation of additional backwinding prior to fitting is not essential.

\section{RESULTS}

The described methodology for the approximation of propagation and characteristic admittance transfer functions has been applied to the line shown in Figure 1, of $150 \mathrm{~km}$ length. The ground resistivity used for fitting is $\rho=100 \Omega \mathrm{m}$.

The estimated poles and residues for the propagation transfer functions are given in Table 1. The error levels can be further reduced by a higher order fitting, however, for each mode, the minimum order realization with practically acceptable error limit has been preferred. The difference between the frequency domain input data and the values of the estimated transfer function is in general very small. For modes 1-4, this difference is insignificant. The most significant difference is observed for modes 5 and 6 , respectively the slowest line mode and the ground mode. The frequency domain fitting of modal propagation transfer functions is given in Figure 4. Figure 5 shows the step response corresponding to mode 6 (the ground mode). The step responses obtained with ground resistivities of $50 \Omega \mathrm{m}$ and $150 \Omega \mathrm{m}$ are shown in the same figure with

Table 1 Estimation results for the modal transfer functions

\begin{tabular}{|c|c|c|c|}
\hline Mode & Poles & Residues & Error $(\%)$ \\
\hline 1 & $\begin{array}{l}-0.8140 \times 10^{8} \\
-0.8396 \times 10^{7} \\
-0.2518 \times 10^{7} \\
-0.7058 \times 10^{6} \\
-0.7982 \times 10^{5}\end{array}$ & $\begin{array}{r}0.8263 \times 10^{7} \\
-0.4603 \times 10^{7} \\
-0.7091 \times 10^{6} \\
-0.1051 \times 10^{6} \\
-0.8613 \times 10^{4} \\
\end{array}$ & 1.13 \\
\hline 2 & $\begin{array}{l}-0.3166 \times 10^{8} \\
-0.5086 \times 10^{7} \\
-0.1776 \times 10^{7} \\
-0.5266 \times 10^{6} \\
-0.5850 \times 10^{5}\end{array}$ & $\begin{array}{r}0.5404 \times 10^{7} \\
-0.3004 \times 10^{7} \\
-0.5822 \times 10^{6} \\
-0.7994 \times 10^{5} \\
-0.5070 \times 10^{4}\end{array}$ & 1.01 \\
\hline 3 & $\begin{array}{c}-0.2264 \times 10^{7} \pm j 0.2115 \times 10^{7} \\
-0.8131 \times 10^{5} \\
-0.7731 \times 10^{6}\end{array}$ & $\begin{array}{c}0.5500 \times 10^{6} \pm j 0.5125 \times 10^{6} \\
-0.9475 \times 10^{4} \\
-0.1048 \times 10^{7}\end{array}$ & 0.91 \\
\hline 4 & $\begin{array}{c}-0.7268 \times 10^{6} \pm j 0.5826 \times 10^{6} \\
-0.3210 \times 10^{5} \\
-0.2823 \times 10^{6}\end{array}$ & $\begin{array}{c}0.2350 \times 10^{6} \pm \mathrm{j} 0.4244 \times 10^{6} \\
-0.2207 \times 10^{4} \\
-0.5319 \times 10^{6}\end{array}$ & 0.74 \\
\hline 5 & $\begin{array}{c}-0.8652 \times 10^{5} \pm j 0.1234 \times 10^{6} \\
-0.5347 \times 10^{5}\end{array}$ & $\begin{array}{c}0.4009 \times 10^{5} \pm j 0.5845 \times 10^{5} \\
-0.1032 \times 10^{6}\end{array}$ & 1.61 \\
\hline 6 & $\begin{array}{c}-0.3295 \times 10^{5} \pm \mathrm{j} 0.3493 \times 10^{5} \\
-0.2486 \times 10^{4} \\
-0.1564 \times 10^{5}\end{array}$ & $\begin{array}{c}0.1132 \times 10^{5} \pm j 0.2003 \times 10^{5} \\
-0.3356 \times 10^{3} \\
-0.2779 \times 10^{5}\end{array}$ & 1.47 \\
\hline
\end{tabular}


dashed lines. The time domain curves show that the accuracy of the estimation is satisfactory.

The step responses in Figures 3 and 5 were obtained from the corresponding state equations using the trapezoidal rule. The calculations were performed with an integration step of $1 \mu$ s. The CPU time with a MIPS-RISC 6280 computer is $0.77 \mathrm{~s}$ for the reduced order model presented in Table 1. Higher order models where each mode is approximated by 10,14 and 20 poles respectively have also been realized for comparison. The RMS errors obtained with these models and the corresponding CPU times for the same simulation conditions are given in Table 2 . This example shows that the use of the reduced order model has resulted in significant savings in the computation time, while the accuracy is within the practically acceptable linits established formerly. Of course, other time

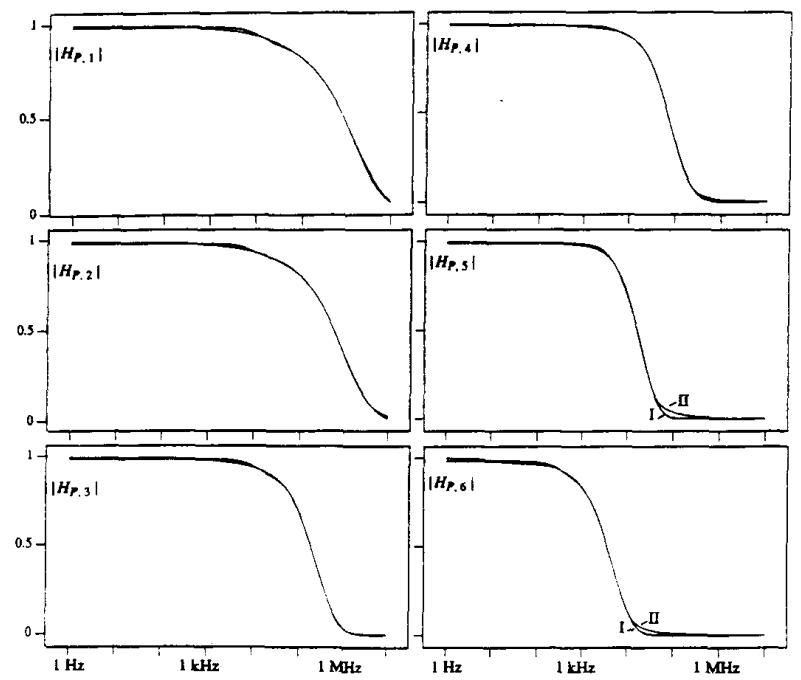

Figure 4 Given (I) and fitted (II) frequency responses for modal propagation functions.

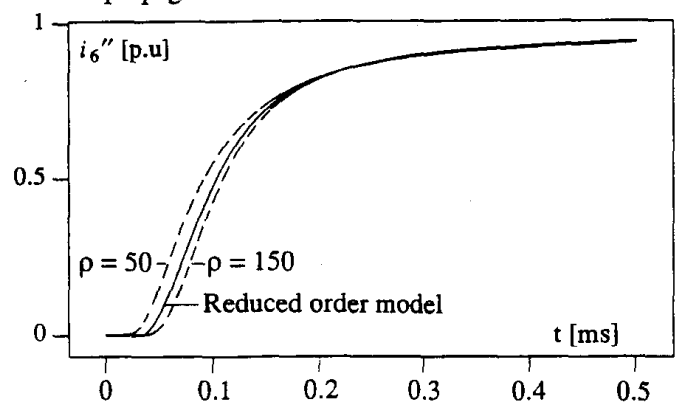

Figure 5 Step response corresponding to mode 6.

Table 2 Errors and CPU times with high order models

\begin{tabular}{|c|c|c|c|c|}
\hline \multirow{2}{*}{ Mode } & \multicolumn{4}{|c|}{ RMS error (\%) } \\
\cline { 2 - 5 } & Red. order & 10 poles & 14 poles & 20 poles \\
\hline 1 & 1.13 & 0.90 & 0.86 & 0.65 \\
\hline 2 & 1.01 & 0.82 & 0.73 & 0.67 \\
\hline 3 & 0.91 & 0.36 & 0.29 & 0.25 \\
\hline 4 & 0.74 & 0.21 & 0.20 & 0.19 \\
\hline 5 & 1.61 & 0.25 & 0.21 & 0.18 \\
\hline 6 & 1.47 & 0.45 & 0.24 & 0.21 \\
\hline \hline CPU time (s) & 0.77 & 1.64 & 2.59 & 4.13 \\
\hline
\end{tabular}

domain simulations may result in smaller relative savings depending on the overhead related to modal/phase transformations and computations pertaining to phenomena at the terminal buses.

The variations of the characteristic admittance for a line mode and the ground mode are shown in Figure 6. The ground resistivity has a bigger effect on the ground mode. The characteristic admittance functions are precisely defined for the line modes (if the transversal conductivity is ignored) in spite of the broad variation of the ground resistivity. An uncertainty, however, exists in the characteristic admittance computed for the ground mode. We can therefore conclude that a larger error limit is acceptable for the ground mode. This result is consistent with the assumptions of Marti in [6]. The variation of the characteristic admittance functions is in general more significant in the low frequency range, particularly below 10 Hz. Practical examples have shown that the order of the fitting depends on the lower limit of the frequency range. In the cases where a long integration time is not required, the characteristic admittance functions can be approximated with a smaller number of poles. Results obtained for the example line (Figure 1) of $150 \mathrm{~km}$ length are given in Table 3 . The estimations are performed over the frequency range $1 \mathrm{~Hz}-1 \mathrm{MHz}$. It can be noted that real poles (as in [5] and [6]) have been sufficient to provide acceptable accuracy in this frequency range. In the cases where a lower frequency range is considered, complex poles may arise. This however does not affect negatively the speed of time domain simulations as these are still performed with real variables.

\section{CONCLUSIONS}

A methodology based on a linear least squares approach is applied for the rational function approximation of the propagation and characteristic admittance transfer functions $\left(H_{p}\right.$ and $\left.Y_{c}\right)$ required in EMTP calculations. Since the estimation technique is not limited to real poles only, as in the conventional EMTP, lower order approximation can be achieved by introducing complex poles when needed.

In any method, high accuracy approximation requires a high model order. This, however, may not be warranted if the data are not very accurate. The precision of the available frequency domain input
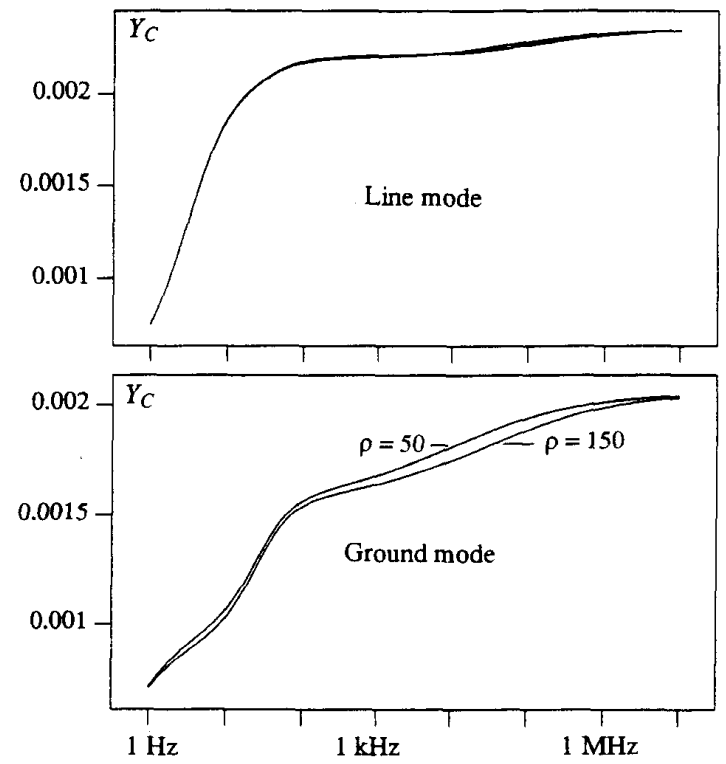

Figure 6 Characteristic admittance functions $Y_{C}$ for a line mode and the ground mode. 
Table 3 Estimation results for the characteristic admittance functions

\begin{tabular}{|c|c|c|c|}
\hline$\overline{\text { Mode }}$ & Poles & Residues & Error (\%) \\
\hline 1 & $\begin{array}{l}-0.8363 \times 10^{4} \\
-0.3683 \times 10^{2} \\
-0.1264 \times 10^{2}\end{array}$ & $\begin{array}{l}-0.2693 \times 10^{0} \\
-0.4893 \times 10^{-1} \\
-0.1812 \times 10^{-1}\end{array}$ & 0.47 \\
\hline 2 & $\begin{array}{l}-0.8713 \times 10^{4} \\
-0.3787 \times 10^{2} \\
-0.1331 \times 10^{2}\end{array}$ & $\begin{array}{l}-0.2809 \times 10^{0} \\
-0.4600 \times 10^{-1} \\
-0.2003 \times 10^{-1}\end{array}$ & 0.52 \\
\hline 3 & $\begin{array}{l}-0.1236 \times 10^{3} \\
-0.4005 \times 10^{2} \\
-0.1401 \times 10^{2}\end{array}$ & $\begin{array}{l}-0.4021 \times 10^{0} \\
-0.3072 \times 10^{-1} \\
-0.2246 \times 10^{-1}\end{array}$ & 0.62 \\
\hline 4 & $\begin{array}{l}-0.8246 \times 10^{5} \\
-0.2334 \times 10^{4} \\
-0.3260 \times 10^{2} \\
-0.1151 \times 10^{2}\end{array}$ & $\begin{array}{l}-0.3915 \times 10^{1} \\
-0.4612 \times 10^{-1} \\
-0.3160 \times 10^{-1} \\
-0.1510 \times 10^{-1}\end{array}$ & 0.60 \\
\hline 5 & $\begin{array}{l}-0.5932 \times 10^{6} \\
-0.5107 \times 10^{5} \\
-0.5120 \times 10^{4} \\
-0.5865 \times 10^{2}\end{array}$ & $\begin{array}{r}-0.7061 \times 10^{2} \\
-0.1335 \times 10^{1} \\
0.5225 \times 10^{-1} \\
-0.3668 \times 10^{-1}\end{array}$ & 0.95 \\
\hline 6 & $\begin{array}{l}-0.7005 \times 10^{6} \\
-0.7916 \times 10^{5} \\
-0.2215 \times 10^{5} \\
-0.4536 \times 10^{4} \\
-0.1520 \times 10^{3}\end{array}$ & $\begin{array}{l}-0.1429 \times 10^{3} \\
-0.7617 \times 10^{1} \\
-0.1599 \times 10^{1} \\
-0.3315 \times 10^{0} \\
-0.9534 \times 10^{-1}\end{array}$ & 1.13 \\
\hline
\end{tabular}

data has therefore been examined in terms of the ground resistivity, the single most important factor which may vary in a broad range in practice. The variation of this parameter affects all modes of the propagation function and the ground mode of the characteristic admittance function. The error levels should, therefore, be determined according to the precision of the input data as reflected in the step responses. It is, however, difficult to specify concrete error tolerances especially in view of the fact that high accuracy is of questionable importance in the fitting of modes strongly affected by the variable and poorly known resistance of the ground return path.

The results presented show that the new methodology for fitting can provide satisfactory accuracy with a small number of real and/or complex poles. The use of lower order models may inprove the efficiency of EMTP calculations by significantly reducing the computation time.

\section{ACKNOWLEDGEMENTS}

Financial support by the Natural Sciences and Engineering Research Council of Canada is gratefully acknowledged. The first author wishes to express his gratitude to Karadeniz Technical University, Turkey, for his sabbatical leave at the University of Toronto.

\section{REFERENCES}

[1] H.W. Dommel, "Digital Computer Solution of Electromagnetic Transients in Single - and Multiphase Networks", IEEE Trans. on Power Apparatus and Systems, Vol. PAS-88 No.4, pp. 388-399, April 1969.

[2] H.W. Dommel and W.S. Meyer, "Computation of Electromagnetic Transients", Proceedings of the IEEE, Vol.62, No.7, pp. 983-993, July 1974.

[3] A. Semlyen and A. Dabuleanu, "Fast and Accurate Switching Transient Calculations on Transmission Lines With Ground Return Using Recursive Convolutions", IEEE Trans. on Power Apparatus and Systems, Vol. PAS-94, pp. 561-571, March/April 1975.

[4] A. Semlyen, "Contributions to the Theory of Calculation of Electromagnetic Transients on Transmission Lines with Frequency Dependent Parameters", IEEE Trans. on Power Apparatus and Systems, Vol. PAS-100, pp. 848-856, February 1981.

[5] J.R. Marti, "Accurate Modeling of Frequency-Dependent Transmission Lines in Electromagnetic Transient Simulations", IEEE Trans. on Power Apparatus and Systems, Vol. PAS-101, pp. 147-157, January 1982.

[6] L. Marti, "Low-Order Approximation of Transmission Line Parameters for Frequency-Dependent Models", IEEE Trans. on Power Apparatus and Systems, Vol. PAS-102, pp. 3582-3589, November 1983.

[7] A.O. Soysal and A. Semlyen, "Practical Transfer Function Estimation and Its Application to Wide Frequency Range Representation of Transformers", IEEE/PES 92 Summer Meeting, Paper No. 92 SM 358-2-PWRD.

A. Oguz Soysal (M'90) was borm in Turkey, in 1951. He received the B.Sc, M.Sc. and Ph.D degrees from Istanbul Technical University. In 1983 he joined ESAS Electrical Industry-power transformer factory as R\&D engineer. In 1986 he was appointed Assistant Professor at Karadeniz Technical University, Turkey. At present, Dr. Soysal is with the same institution as Associate Professor, back from his sabbatical leave at the University of Toronto. His research areas include analysis and control of power systems, power transmission and distribution, study of electromagnetic transients and system identification.

Adam Semlyen (F'88) was bom and educated in Rumania where he obtained a Dipl. Ing. degree and his $\mathrm{Ph} . \mathrm{D}$. He started his career with an electric power utility and held an academic position at the Polytechnic Institute of Timisoara, Rumania. In 1969 he joined the University of Toronto where he is a professor in the Department of Electrical and Computer Engineering, emeritus since 1988. His research interests include the steady state and dynamic analysis of power systems, electromagnetic transients, and power system optimization. 